\title{
Estimating Attenuation of Ultraviolet Radiation in Streams: Field and Laboratory Methods
}

\author{
Patrick Belmont ${ }^{\star 1}$, Bruce R. Hargreaves ${ }^{1}$, Donald P. Morris ${ }^{1}$ and Craig E. Williamson ${ }^{2}$ \\ ${ }^{1}$ Earth and Environmental Science Department, Lehigh University, Bethlehem, PA \\ ${ }^{2}$ Department of Zoology, Miami University, Oxford, OH \\ Received 2 February 2007; accepted 30 April 2007; Online Early; DOI: 10.1111/j.1751-1097.2007.00164.x
}

\begin{abstract}
We adapted and tested a laboratory quantitative filter pad method and field-based microcosm method for estimating diffuse attenuation coefficients $\left(K_{\mathrm{d}}\right)$ of ultraviolet radiation (UVR) for a wide range of stream optical environments $\left(K_{\mathrm{d} 320}=3-44 \mathrm{~m}^{-1}\right)$. Logistical difficulties of direct measurements of UVR attenuation have inhibited widespread monitoring of this important parameter in streams. Suspended sediment concentrations were manipulated in a microcosm, which was used to obtain direct measurements of diffuse attenuation. Dissolved and particulate absorption measurements of samples from the microcosm experiments were used to calibrate the laboratory method. Conditions sampled cover a range of suspended sediment (0-50 mg $\mathrm{L}^{-1}$ ) and dissolved organic carbon concentrations (1-4 mg $\mathrm{L}^{-1}$ ). We evaluated four models for precision and reproducibility in calculating particulate absorption and the optimal model was used in an empirical approach to estimate diffuse attenuation coefficients from total absorption coefficients. We field-tested the laboratory method by comparing laboratory-estimated and field-measured diffuse attenuation coefficients for seven sites on the main stem and $\mathbf{1 0}$ tributaries of the Lehigh River, eastern Pennsylvania, USA. The laboratory-based method described here affords widespread application, which will further our understanding of how stream optical environments vary spatially and temporally and consequently influence ecological processes in streams.
\end{abstract}

\section{INTRODUCTION}

Ultraviolet radiation (UVR) plays an important role in aquatic ecosystems $(1,2)$. Much theoretical and empirical work has been dedicated to describing optics in marine and freshwater lentic environments, which has resulted in a significant literature demonstrating the influence of UVR in such environments (3), but relatively little optical work has been performed in freshwater lotic systems. Because the optical environment in streams is closely linked to processes occurring throughout the watershed, it is likely that large-scale human alteration of landscapes $(4,5)$, watershed hydrology and climate $(6,7)$ have significant influences on light transparency. In addition, destruction of stratospheric ozone (8) is increasing incident UVR in stream ecosystems with unknown consequences

*Corresponding author email: pwb3@Lehigh.edu (Patrick Belmont) (c) 2007 The Authors. Journal Compilation. The American Society of Photobiology 0031-8655/07 for these shallow, often highly exposed, lotic ecosystems (9). Short-term, laboratory-based studies of organismal response to UVR exposure are inadequate for understanding the full effects of UVR on aquatic organisms in the natural setting $(10,11)$.

The lack of work focused on UVR in stream ecosystems is, in part, because of difficulty of measuring UVR attenuation directly in stream ecosystems, and the lack of alternative methods for estimating UVR attenuation from proxy measurements. Direct measurement of UVR attenuation in streams is difficult and in many cases impractical. Logistical complications, including expense and bulkiness of the necessary instruments, necessary depth for profile measurements ( $\sim 0.5 \mathrm{~m}$ even with the smallest radiometers) and sensitivity of the instrument to verticality and surface smoothness make in situ measurements of UVR diffuse attenuation ( $\left.K_{\mathrm{dUV}}\right)$ highly impractical for many ecological applications. Yet, to study the ecological implications of UVR exposure it is imperative to know, within reasonable error, the penetration of UVR through the water column. Furthermore, our field observation indicates that the temporal variability in stream transparency is substantial, suggesting that comparative watershed studies would benefit from an approach that lends itself to more frequent sampling intervals. A quantitative filter pad technique (QFT, 12-14) has been developed to account for particulate absorption in marine environments and has been applied to UV wavelengths for numerous lake studies (15-17). This procedure, with proper calibration, should allow for laboratory measurement of particulate absorption of stream sediments, which could then be used to reconstruct total absorption and diffuse attenuation in the natural water column. The primary benefits of using the QFT approach include (1) ease of processing large sample batches, potentially collected simultaneously, (2) avoiding logistical complications of performing in situ measurements, and (3) independently modeling water column optics from inherent optical properties, after which any other influences (canopy cover, sky conditions, stream orientation, etc.) can be appropriately accounted for over entire stream stretches.

The objectives of this study were two-fold: (1) to develop a microcosm approach for measuring $K_{\mathrm{dUV}}$ using a profiling radiometer when turbulent or rapid flow, shallow depth, or obstructed sky conditions, make direct in situ measurement impractical, and (2) develop a method for estimating $K_{\mathrm{dUV}}$ in streams from several types of laboratory measurements of 
stream water samples. Here we discuss the development, limitations and potential applications of these methods and techniques to investigations of lotic systems.

\section{MATERIALS AND METHODS}

Chemical methods. All samples were filtered using Whatman glass fiber filters $(\mathrm{GF} / \mathrm{F})$ with a minimum retention size of $\sim 0.7 \mu \mathrm{m}$. Therefore, dissolved matter is operationally defined as anything smaller than $0.7 \mu \mathrm{m}$ and all measurements (optical or chemical) are consistent with that definition. Total suspended solids (TSS) were determined by filtering a measured volume of sample through a preweighed, ashed $\mathrm{GF} / \mathrm{F}$ filter $\left(450^{\circ} \mathrm{C}\right.$ for $\left.3 \mathrm{~h}\right)$ and drying to a constant weight at $65^{\circ} \mathrm{C}$. Loss-on-ignition was determined by combusting the filters at $450^{\circ} \mathrm{C}$ for $3 \mathrm{~h}$. Percent particulate organic carbon (POC) was calculated as $47 \%$ (18) of dry-weight loss-on-ignition. Dissolved organic carbon (DOC) concentration in the GF/F filtrate was measured using a Shimadzu TOC 5000 after acidification of the sample to $\mathrm{pH}$ below 2.0.

Microcosm estimates of diffuse attenuation coefficients. A microcosm was constructed which allowed for the estimation of $K_{\mathrm{dUV}}$ in circumstances where direct in situ measurement in streams was impractical. The utility of the microcosm was verified by comparing measurements made in situ (under suitable conditions) with those performed in the device (see below). Additionally, measurements made in the microcosm were compared with laboratory measurements of particulate, dissolved and total absorption.

The microcosm was constructed of UV-transparent OP-4 acrylic (CYRO Industries; $272 \mathrm{~nm}$ cutoff) with dimensions $(40 \mathrm{~cm} \times 40 \mathrm{~cm} \times$ $20 \mathrm{~cm}$ ), which was supported on wooden legs. Plywood was used to shade upwelling light from the ground below the microcosm (Fig. 1).

All light measurements in the microcosm were obtained using a PUV-501 (Biospherical Instruments, Inc., San Diego, CA) profiling UV radiometer with cosine response and five optical channels (305, $320, \quad 340, \quad 380 \mathrm{~nm}$ having $8-10 \mathrm{~nm}$ bandwidth and $400-700 \mathrm{~nm}$ photosynthetically available radiation (PAR); 19), which was held in place by clamps directly in contact with the bottom of the clear acrylic box.

Average downwelling irradiance $\left(E_{\mathrm{d}}\right)$ at two depths in the microcosm was calculated from a minimum of 120 measurements (at $1 \mathrm{~s}$ intervals) at each depth. Attenuation coefficients in the microcosm were calculated as:

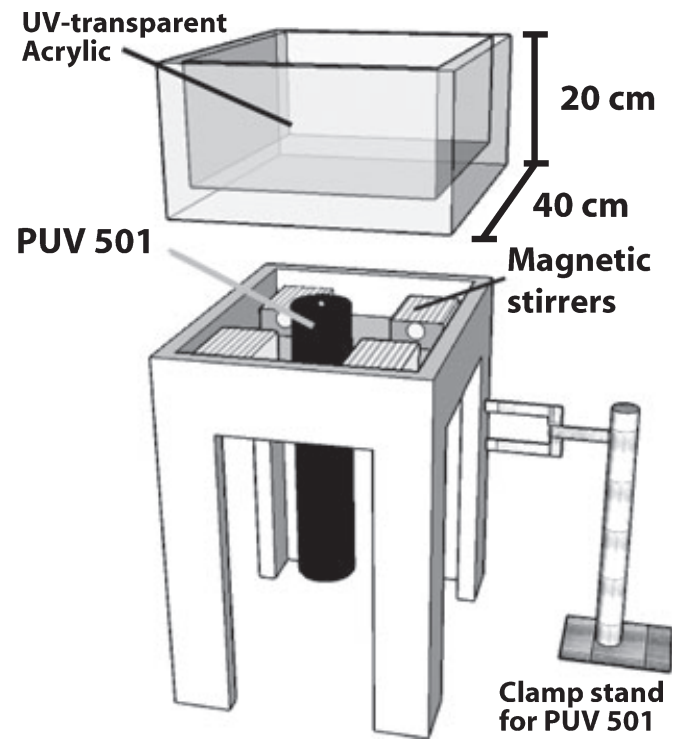

Figure 1. Microcosm assembly using UV-transparent OP-4 acrylic and a PUV-501 radiometer. The radiometer and four magnetic stirrers were in contact with the bottom of the acrylic box during the experiments.

$$
K_{\mathrm{d}}=\frac{\ln \left\{\left(E_{\mathrm{d}_{1}} / E_{\mathrm{d}_{2}}\right)\right\}}{d_{2}-d_{1}}
$$

where $E_{\mathrm{d}_{1}}$ is downwelling irradiance $\left(\mu \mathrm{W} \mathrm{cm} \mathrm{cm}^{-2} \mathrm{~nm}^{-1}\right)$ at the shallow depth $\left(d_{1}\right.$, in $\left.\mathrm{m}\right)$ and $E_{\mathrm{d} 2}$ is the downwelling irradiance at the deeper depth $\left(d_{2}\right)$.

Microcosm measurements of attenuation were made within $2 \mathrm{~h}$ of solar noon on 9 November 2003, 21 November 2003 and 5 April 2005, under clear, open sky conditions. Water used for the three microcosm experiments was taken from the Lehigh River and Saucon Creek, a fourth-order tributary to the Lehigh, in Bethlehem, PA. After validation of the approach, several water samples were manipulated to provide varying rates of attenuation by dissolved and particulate fractions. This was done by adding specific proportions of a sediment concentrate to water that had been previously filtered. This concentrate was obtained by allowing water samples, taken from the Lehigh River and Saucon Creek during high-flow conditions, to settle for approximately 3 days in 5 gallon jugs and decanting off the top fraction. Magnetic stirrers were used to maintain the particles in suspension in the microcosm. Samples for chemical and optical analysis were collected from the microcosm during each trial of each experiment and stored in acid-washed polypropylene bottles and processed within $24 \mathrm{~h}$.

Comparison of microcosm and in situ measurements of diffuse attenuation coefficients. Validation of the microcosm as a means of estimating diffuse attenuation was accomplished by directly measuring $K_{\mathrm{d}}$ in situ and repeating the measurements in the field using the microcosm (i.e. near simultaneous measurements using the two techniques under similar sky conditions). This comparison was performed at Lake Nockamixon, Bucks County, PA $\left(40.470^{\circ} \mathrm{N}\right.$, $75.187^{\circ} \mathrm{W}, 120 \mathrm{~m}$ above sea level) within $2 \mathrm{~h}$ of solar noon on 24 October 2003, under clear, blue sky conditions. Three separate lake profiles were performed using the PUV-501 (which has a depth sensor with $0.4 \mathrm{~cm}$ resolution). Values of $K_{\mathrm{d}}$ were calculated from the slope of the regression of the downward welling irradiance $\left(E_{\mathrm{d}}\right)$ versus depth $(z)$. A lake (rather than a stream) was selected for this comparison because it provided the most favorable conditions for in situ measurement of $K_{\mathrm{d}}$ available.

Direct measurement of diffuse attenuation coefficients in streams and rivers. Direct measurement of $K_{\mathrm{d}}$ was performed on 2 June and 4 June 2004, at several sites along the main stem of the Lehigh River and several of its largest tributaries. Three replicate measurements were made at each site using a BIC submersible UV-PAR radiometer (manufactured and solar calibrated by Biospherical Instruments, Inc., San Diego, CA) with the same wavelength sensors as the PUV 500 except that there was no $340 \mathrm{~nm}$ sensor. Care was taken to minimize canopy cover at field sites, but this was sometimes unavoidable, in which case constant canopy shade conditions were chosen to ensure uniformity of incident light at the sampling site.

Measurements of dissolved absorption $\left(a_{\mathrm{d}}\right)$ in the laboratory. Measurements of dissolved absorption $\left(a_{\mathrm{d}}\right)$ were made on filtrate from Whatman glass fiber filters (GF/F). Measurements were made using a Shimadzu UV-VIS 1601 dual beam spectrophotometer with a $10 \mathrm{~cm}$ quartz Supersil ${ }^{\mathrm{TM}}$ cuvette referenced to air. To calculate dissolved spectral absorption $\left(a_{\mathrm{d}}\right)$, we subtracted the spectral optical density of deionized water $\left(A_{\mathrm{di}}\right)$, measured with each sample batch, from the sample absorption $\left(A_{\mathrm{d}}\right)$ spectrum $(800-200 \mathrm{~nm})$ :

$$
\begin{gathered}
a_{\mathrm{d}}(\lambda)=\left(A_{\mathrm{d}}(\lambda)-A_{\mathrm{di}}(\lambda)\right) \times d_{\mathrm{p}}-A_{775-800} \\
\text { where } d_{\mathrm{p}}=(L N(10) \times 100 / L)
\end{gathered}
$$

where $d_{\mathrm{p}}$ accounts for the length of the cuvette ( $L$ is the length of the cuvette in centimeters) and converts the logarithm of OD from base 10 to base e. $A_{775-800}$ is a baseline offset calculated as the average optical density value measured between 775 and $800 \mathrm{~nm}$. The baseline offset is necessary for dissolved absorption spectra because actual absorption by chromophoric dissolved organic matter (CDOM) is negligible in these wavelengths. Therefore, any change in light transmission detected by the spectrophotometer is actually due to instrument drift and scattering in the solution, which was assumed to be spectrally flat and was subtracted from the entire spectrum. Dissolved absorption coefficients were then calculated as 10 point averages centered on the wavelength of interest. Estimates of the absorption coefficient of pure water $\left(a_{\mathrm{w}}\right)$ were subtracted from those obtained for the samples. These 
Table 1. Values used for the absorption of pure water in UV-A and UV-B wavelengths (B. R. Hargreaves, unpublished).

\begin{tabular}{lc}
\hline Wavelength $(\mathrm{nm})$ & $a_{\mathrm{w}}\left(\mathrm{m}^{-1}\right)$ \\
\hline 380 & 0.007 \\
340 & 0.016 \\
320 & 0.028 \\
305 & 0.043
\end{tabular}

estimates (Table 1) were first derived from an extension of a model published by Pope and Fry (20) and then calibrated in an ultratransparent lake by Hargreaves (unpublished).

Measurement of particulate absorption $\left(a_{\mathrm{p}}\right)$ in the laboratory. Measurements of particulate absorption $\left(a_{\mathrm{p}}\right)$ were performed with a modified version of the QFT using a Shimadzu UV-VIS 1601 dual beam spectrophotometer. This method uses a $25 \mathrm{~mm}$ Whatman GF/F filter (22 $\mathrm{mm}$ effective diameter in filter tower) for concentration of particulate material, measured against a clean, wet Whatman GF/A filter (rinsed with deionized water) as a reference. Both filters are similar in construction; the advantage of using the GF/A as the reference is in its higher transmittance.

Approximately $50 \mathrm{~mL}$ of deionized water was passed through each filter using vacuum filtration prior to sample filtration. Care was taken to ensure that the filters were kept wet during and after filtration. Quartz disks were inserted over the receiving sensors of the spectrophotometer, to which the wet filters readily adhered. Before placing the filters on the quartz disks, the instrument was zeroed across the entire spectrum of interest $(800-280 \mathrm{~nm})$ by running a baseline correction program. The optical density of the rinsed GF/F filter was then measured $(800-280 \mathrm{~nm})$ relative to the GF/A filter. This serves as a "baseline scan." The GF/F filter was then removed from the spectrophotometer and used to collect particulate material from a measured volume of sample $(25-250 \mathrm{~mL})$. The sample volume was varied to allow a minimal amount of particulate material to be spread evenly over the filter. The spectral optical density was then measured again, relative to the same GF/A filter. This procedure was repeated after adding incrementally small amounts of particulate material to the same filter. Care was taken to maintain an optical density below an instrument value of $\sim 1.0$, but this value will likely vary depending on the capabilities of individual instruments. Measurement of particulate absorption is time sensitive as microbial degradation may alter its optical properties. Therefore, we refrigerated all samples and measurements were completed within $24-48 \mathrm{~h}$ of collection. To investigate the effect of the amount of water filtered on the transparency of the filters, we sequentially filtered, in additive fashion, 50, 100, 200, 300, 500 and $800 \mathrm{~mL}$ of deionized water through the same GF/F filter and measured its spectral optical density against a GF/A reference. The effect of reference filter desiccation was investigated by measuring the optical density of a $47 \mathrm{~mm} \mathrm{GF} / A$ filter against air at $<1,3,6,9,12,30,45$ and $60 \mathrm{~min}$ after filtering the standard $50 \mathrm{~mL}$ of water through it.

Several calculations were necessary to convert the optical density measured with the spectrophotometer to the particulate absorption coefficient $\left(a_{\mathrm{p}}(\lambda)\right.$, hereafter simply referred to as $\left.a_{\mathrm{p}}\right)$. Raw optical density spectra $(800-280 \mathrm{~nm})$ for the baseline scan (GF/F to GF/A) and at least two sequential volumes of sample were required to obtain reliable data. The spectra were first smoothed using a 10-point running average.

There is an active debate regarding the best method to correct particulate optical density for the path length amplification that inherently occurs as a result of multiple scattering as light propagates through the sample/filter matrix. A thorough discussion regarding approaches to account for this phenomenon can be found in Roesler (13) and Lohrenz (14) and the interactive roles of reflectance and transmittance in QFT estimates of absorption are discussed in Tassan and Ferrari (21-23). In addition, different volumes of sample filtered through the filter pad should, ideally, yield the same particulate absorption coefficient, once path length amplification and the volume filtered have been taken into consideration. In practice, however, slightly different results are typically observed, regardless of the equations used for calculation. Larger volumes typically yield lower values of $a_{\mathrm{p}}$. The likely mechanism for this is that layering of particles on the filter pad may effectively "shade" some particles or alter the amount of reflectance relative to absorbance. To determine the best way to deal with these observed problems, we tested several different methods for calculation of the particulate absorption coefficient.

We tested two general models to calculate $a_{\mathrm{p}}$ from optical density measurements using the QFT. The first model is taken from Lohrenz (14):

$$
\begin{aligned}
& a_{\mathrm{p}}(\lambda)=\frac{A_{\mathrm{fp}}(\lambda)}{\left(\beta \times d_{\mathrm{g}} \times\left(1-A_{\mathrm{fp}}(\lambda)\right)\right)} \\
& \text { where } A_{\mathrm{fp}}(\lambda)=1-10^{-\left(\mathrm{OD}_{\mathrm{f}}(\lambda)\right)}
\end{aligned}
$$

where $A_{\mathrm{fp}}$ is the absorbance of the filter loaded with particles, $\beta$ is the path length amplification factor, $\operatorname{OD}_{\mathrm{f}}(\lambda)$ is the optical density of the filter with sample retained minus the baseline scan and the geometric path length $\left(d_{\mathrm{g}}\right.$, calculated as the volume filtered divided by the effective filter area) is in meters. Another approach is to calculate $a_{\mathrm{p}}$ with a linear equation, similar to the approach of Roesler (13):

$$
\begin{aligned}
& a_{\mathrm{p}}(\lambda)=\left(\frac{1}{\beta}\right) \times p \times \operatorname{OD}_{\mathrm{f}}(\lambda) \\
& \text { where } p=L N(10) \times\left(\frac{100}{d_{\mathrm{g}}}\right)
\end{aligned}
$$

where the geometric path length is in centimeters.

Laboratory estimates of $K_{\mathrm{d}}(\lambda)$. The sum of $a_{\mathrm{w}}, a_{\mathrm{d}}$ and $a_{\mathrm{p}}$ is the total absorption coefficient $\left(a_{\mathrm{t}}\right)$, which quantifies beam absorption. One further adjustment must be made to extrapolate this number to $K_{\mathrm{d}}(\lambda)$, which quantifies the in situ attenuation of diffuse light. $K_{\mathrm{d}}(\lambda)$ is an "apparent optical property" because the actual magnitude for any given time or location is dependant on the structure of the incident light field. The correction factor that accounts for this is referred to as the mean cosine of the angle of photons from the vertical, $\mu$. In nature $\mu$ is dynamic, as numerous environmental variables affect the angular distribution of natural light, including scattering in the water column. For purposes of ecological modeling, however, it is necessary to approximate a mean $\mu$ factor, with the understanding that actual values will differ slightly, and our estimate of $\mu$ will include both the influence of scattering and diffuse light on the relation between $K_{\mathrm{d}}$ and total absorption. The diffuse attenuation coefficient and total absorption coefficient are related as:

$$
K_{\mathrm{d}}(\lambda)=\frac{a_{\mathrm{t}}(\lambda)}{\mu(\lambda)}
$$

By calculating $a_{\mathrm{t}}(\lambda)$ and $K_{\mathrm{d}}(\lambda)$ from measurements in the laboratory and microcosm, respectively, we can solve (Eq. 8) for the mean cosine $(\mu(\lambda))$. Any $\mu(\lambda)$ values that originally exceeded 1.0 due to measurement error, were reduced to 1.0 for the purposes of calculating $K_{\mathrm{d}}(\lambda)$ values for model development.

We evaluated each of the four calculation models (Lohrenz with $\beta=2.0$, Lohrenz with $\beta=2.7$, Roesler with $\beta=2.0$ and Roesler with $\beta=2.7$ ) using four general criteria. The first criterion was the regression slope of the $K_{\mathrm{d}}(\lambda)$ estimated from laboratory measurements against the $K_{\mathrm{d}}(\lambda)$ measured in the microcosm. Slopes closest to 1 represent the best agreement across the range of optical environments. Second, we evaluated each of the $\mu(\lambda)$ values generated using a variant of (Eq. 8), rejecting any that exceed 1.0. Third, we compared the measurements of $a_{\mathrm{p}}(\lambda)$ and $a_{\mathrm{t}}(\lambda)$ made using an integrating sphere (model RSA-SZ-16; Labsphere, North Sutton, NH, installed in a Shimadzu UV160U spectrophotometer, which allows for placement of a $1 \mathrm{~cm}$ cuvette at the transmittance port with a spectralon plug covering the reflectance port) with those calculated with the QFT. Lastly, we evaluated the reproducibility of $a_{\mathrm{p}}(\lambda)$ among different volumes filtered for each of the models.

\section{RESULTS}

\section{Optimization of our QTF method}

We used regression analysis to investigate three possible baseline techniques for our QTF method. Three trials of each technique were detrended in $10 \mathrm{~nm}$ bands in the blue, UV-A 
Table 2. The sum of squared residuals from $10 \mathrm{~nm}$ bands in the blue, UV-A and UV-B regions demonstrating the amount of "noise" in the baseline scan signal.

\begin{tabular}{lccc}
\hline & GF/F-GF/A & GF/F-GF/F & GF/F-air \\
\hline Blue (445-455 nm) & 0.000070 & 0.000096 & 0.000547 \\
UV-A (375-385 nm) & 0.000056 & 0.000083 & 0.000256 \\
UV-B (310-320 nm) & 0.000378 & 0.000423 & 0.002066
\end{tabular}

and UV-B regions and used to calculate the sum of squared residuals. The variance caused by instrument sensitivity was minimized for all blue and UVR wavelengths using a combination of a $\mathrm{GF} / \mathrm{F}$ filter used for sample loading measured against a GF/A reference filter yield. Higher variance was observed using a $\mathrm{GF} / \mathrm{F}$ referenced to another $\mathrm{GF} / \mathrm{F}$ or air (Table 2). The explanation for this is related to the intermediate optical density of GF/A filters. An intermediate reference decreases the large disparity in light transmission generated when measuring a $\mathrm{GF} / \mathrm{F}$ versus air, while still maintaining a relatively strong reference beam, which is much diminished when referencing against another GF/F.

The variability of $\mathrm{GF} / \mathrm{F}$ filter baselines was measured for 184 arbitrarily selected scans against GF/A reference covering many different filter batches over the course of 3 years. The coefficient of variation is $\sim 10 \%$ or 0.025 absorption units for UVR wavelengths, though it should be noted that this factor is minimized by obtaining a $\mathrm{GF} / \mathrm{F}$ to $\mathrm{GF} / \mathrm{A}$ baseline scan for every sample.

Measuring the optical density of a GF/A filter against air multiple times over a $60 \mathrm{~min}$ interval (without rewetting) yielded variation of less than $1 \%$. Rewetting the reference filter by dipping it in a watch glass filled with deionized water every $\sim 30$ min further minimizes variability. After the initial filtration of $50 \mathrm{~mL}$ of deionized water, the optical density of the $\mathrm{GF} / \mathrm{F}$ filter was unchanged by the passage of an additional $750 \mathrm{~mL}$ of deionized water (this amount was more than sufficient to perform particulate absorption scans on our stream samples).

Particulate absorption spectra of highly organic samples from our streams (e.g. peat samples with LOI $>85 \%$ ) indicated substantial absorption at long wavelengths (contrary to the pervasive assumption in the aquatic optics community that particles primarily scatter at these wavelengths). This suggests that a long-wavelength null-point correction (i.e. subtracting optical density at $750 \mathrm{~nm}$ from the entire spectrum) is not always appropriate. The need for such a correction can be argued for some samples that deviate from the expected linear trend on a semi-log plot of $a_{\mathrm{p}}$ and wavelength, though it typically does not change the particulate absorption coefficient significantly.

In some studies, glass fiber filters are ashed prior to use. We compared the variability of spectra between three ashed and three non-ashed GF/F filters referenced against GF/A, with and without sample loaded onto each of the filters. Ashed filters exhibited 2- to 10-fold higher standard deviations compared to non-ashed filters.

\section{Comparison of microcosm and in situ measurements of diffuse attenuation coefficients}

The attenuation measurements made in the microcosm and in Lake Nockamixon yield highly comparable results (Table 3).
Table 3. Mean $K_{\mathrm{d}}$ values for three lake profiles and three trials in the microcosm.

\begin{tabular}{lcccc}
\hline & $\begin{array}{c}\text { In situ } \\
K_{\mathrm{d}}\left(\mathrm{m}^{-1}\right)\end{array}$ & $\begin{array}{c}2 \sigma \\
\mathrm{SE}\end{array}$ & $\begin{array}{c}\text { Microcosm } \\
K_{\mathrm{d}}\left(\mathrm{m}^{-1}\right)\end{array}$ & $\begin{array}{l}2 \sigma \\
\mathrm{SE}\end{array}$ \\
\hline PAR & 2.1 & \pm 0.03 & 2.3 & \pm 0.12 \\
380 & 11.6 & \pm 0.35 & 11.3 & \pm 0.31 \\
340 & 19.3 & \pm 0.41 & 18.9 & \pm 0.54 \\
320 & 24.8 & \pm 0.62 & 24.5 & \pm 0.65 \\
$305^{*}$ & 29.8 & NA & 30.9 & \pm 0.07 \\
& & & & \\
\hline
\end{tabular}

*Only one of the lake profiles and two of the microcosm trials were suitable for estimation of $K_{\mathrm{d} 305}$, hence standard error could not be calculated for lake $K_{\mathrm{d} 305}$ and $n=2$ for microcosm $K_{\mathrm{d} 305}$.

Comparison of mean values indicates agreement within $\pm 4 \%$ for all UV wavelengths. At lower values of $K_{\mathrm{d}}$ it became increasingly difficult to measure transparency in the $20 \mathrm{~cm}$ path length of the microcosm. Our microcosm estimates of $K_{\mathrm{d}}$ for PAR $\left(K_{\mathrm{dPAR}} \sim 2 \mathrm{~m}^{-1}\right)$ varied by about $\pm 8 \%$ from direct in situ measurements and the standard error was about four times higher.

We attempted to perform a comparison of the microcosm to direct in situ measurements of $K_{\mathrm{d}}$ in highly transparent Dutch Springs, PA $\left(K_{\mathrm{d} 320} \sim 0.5 \mathrm{~m}^{-1}\right)$ but were unsuccessful because the resolution of light measurements was not sufficient in the limited path length of the microcosm. At such low attenuation, the resolution of the microcosm is highly sensitive to environmental variables such as wind and sky conditions. Given the variability of the microcosm measurements at Dutch Springs, we estimate that the container would need to be $\sim 70 \mathrm{~cm}$ deep to obtain statistically distinct (3 standard deviations) values of $E_{\mathrm{d} 320}$ under similar sky and wind conditions, when $K_{\mathrm{d} 320}$ is $0.5 \mathrm{~m}^{-1}$. Gusty winds disrupting the water surface during measurements at Dutch Springs contributed substantially to the poor resolution. The lowest reliable $K_{\mathrm{d} 320}$ measured with the microcosm was 2.7 , but we expect that $K_{\mathrm{d} 320}$ values around 1.0 could be reliably measured using the apparatus, as described here, under ideal environmental conditions.

\section{Comparison of $K_{d}$ estimates made in the microcosm to those made in the laboratory using the spectrophotometer}

Three microcosm experiments were performed in which the concentration of suspended particulate material was varied over a range that we expect covers most lotic environments $\left(0-80 \mathrm{mg} \mathrm{L}^{-1}\right)$. The concentration of DOC varied between experiments (1-4 mg C L ${ }^{-1}$ ) but was held constant for each of the trials of a single experiment as the concentration of suspended particulate material was increased. $K_{\mathrm{d}}$ was measured in the microcosm (via the PUV-501) for each of the trials, then samples were collected for laboratory estimation of $K_{\mathrm{d}}$ in the spectrophotometer (from $a_{\mathrm{p}}$ and $a_{\mathrm{d}}$ measurements).

$K_{\mathrm{d}}$ values obtained in this set of experiments covers much of the range expected in stream environments where UVR is likely to be an important factor $\left(1-29 \mathrm{~m}^{-1}\right.$ for $380 \mathrm{~nm}$; $2-38 \mathrm{~m}^{-1}$ for $340 \mathrm{~nm} ; 3-44 \mathrm{~m}^{-1}$ for $\left.320 \mathrm{~nm}\right)$. Two replicate microcosm measurements of $K_{\mathrm{d}}$ were performed for each of the three experiments to quantify the precision of the method across the entire range of attenuation values. The coefficient of variation for the six sets of replicate measurements of $K_{\mathrm{d}}$ was $14 \%(380 \mathrm{~nm}), 9 \%(340 \mathrm{~nm})$ and $8 \%(320 \mathrm{~nm})$. Notice that 
Table 4. Average $\mu$ factors $( \pm 1 \sigma \mathrm{SE})$ calculated from all experimental trials for each of the models ( $n=23$ for 380,340 and $320 \mathrm{~nm}, n=16$ for $305 \mathrm{~nm}$ ).

\begin{tabular}{lcccc}
\hline $\begin{array}{l}\text { Wavelength } \\
(\mathrm{nm})\end{array}$ & Roesler 2.0 & Roesler 2.7 & Lohrenz 2.0 & Lohrenz 2.7 \\
\hline 380 & $0.76 \pm 0.06$ & $0.69 \pm 0.06$ & $0.89 \pm 0.06$ & $0.78 \pm 0.06$ \\
340 & $0.82 \pm 0.05$ & $0.76 \pm 0.05$ & $0.96 \pm 0.05$ & $0.86 \pm 0.04$ \\
320 & $0.85 \pm 0.04$ & $0.79 \pm 0.04$ & $0.99 \pm 0.04$ & $0.90 \pm 0.04$ \\
305 & $0.96 \pm 0.05$ & $0.90 \pm 0.05$ & $1.09 \pm 0.04$ & $1.00 \pm 0.05$
\end{tabular}

the variation decreases with increasing wavelength (and increasing $K_{\mathrm{d}}$ ). This variability is most certainly a high estimate for the method since three of the six replicates had relatively low sample attenuation (i.e. $K_{\mathrm{d} 340}<4 \mathrm{~m}^{-1}$ ).

Samples from each of the trials performed in the microcosms were analyzed spectrophotometrically in the laboratory for $a_{\mathrm{d}}$ and $a_{\mathrm{p}}$ using the modified QFT. Average $\mu$ factors were empirically derived as the mean value of $K_{\mathrm{d}} / a_{\mathrm{t}}$ for the Nockamixon trials and all trials in microcosm experiments 2 and 3 and are shown in Table 4.

Reasonable values for $\mu$ must be less than 1.0 because beam absorption is inherently lower than attenuation of diffuse light. The $\mu$ value for $305 \mathrm{~nm}$ light calculated from the Lohrenz 2.7 model may be reasonable considering the error associated with measurements, but the Lohrenz 2.0 model appears to be unreasonably high.

Regression analysis of $K_{\mathrm{d}}$ calculated in the laboratory using spectrophotometric methods versus those measured in the microcosm provides a highly significant correlation for UVR wavelengths (Fig. 2) over the wide range of suspended sediment concentrations. The slope of each regression indicates the degree of correlation between laboratory and microcosm estimates of attenuation coefficients (a slope of 1.0 indicates perfect agreement). The $95 \%$ confidence intervals on regression slopes for each of the calculation methods include the value 1.0. The $r^{2}$ value is a measure of the error across the entire gradient of attenuation values.

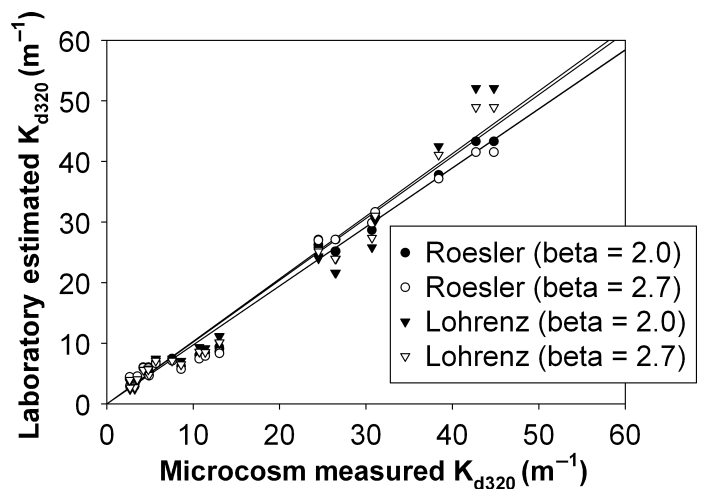

Figure 2. Strong correlation of laboratory-estimated and microcosmmeasured attenuation coefficients $\left(K_{\mathrm{d} 320}\right)$ using each of the four calculation models. Regression slopes were $0.974,0.973,1.033$ and 1.021 for the Roesler $(\beta=2.0)$, Roesler $(\beta=2.7)$, Lohrenz $(\beta=2.0)$ and Lohrenz $(\beta=2.7)$ models, respectively. The $r^{2}$ values are 0.983 , $0.975,0.953$ and 0.974 for the Roesler $(\beta=2.0)$, Roesler $(\beta=2.7)$, Lohrenz $(\beta=2.0)$ and Lohrenz $(\beta=2.7)$ models, respectively. All regressions were forced through zero, though offset from zero on the $y$-intercept for each of the regressions was less than $1.5 \mathrm{~m}^{-1}$ when not forced through zero.
On two occasions an integrating sphere was used along with the QFT method to help identify the appropriate path length amplification factor for our fluvial samples. The total absorption coefficient of undiluted Lehigh River water was measured using the integrating sphere and then compared to the total absorption coefficient calculated using the QFT. Both approaches were then repeated on diluted samples (1:1 using sample filtrate). Total absorption coefficients measured in the integrating sphere were $9.0 \mathrm{~m}^{-1}(380 \mathrm{~nm})$ and $20.9 \mathrm{~m}^{-1}(305 \mathrm{~nm})$ for whole water, and $7.9 \mathrm{~m}^{-1}(380 \mathrm{~nm})$ and $18.0 \mathrm{~m}^{-1}(320 \mathrm{~nm})$ for the diluted sample. Particulate absorption coefficients estimated via the QTF method were calculated using the Lohrenz and the Roesler and the commonly published $\beta$ values for each of the models discussed above.

The percent deviation in $a_{\mathrm{t}}$ obtained using the QFT method compared to $a_{\mathrm{t}}$ measured in the integrating sphere was smallest using the Lohrenz approach with a $\beta$ factor of 2.7 (Fig. 3a). In contrast, the percentage deviation between the QFT values of $a_{\mathrm{p}}$ compared to back-calculated integrating sphere estimates of $a_{\mathrm{p}}$, [calculated by subtracting $\mathrm{a}_{\mathrm{d}}(\lambda)$ from measured $a_{\mathrm{t}}(\lambda)$ ] was minimized when using the Roesler approach and a $\beta$ factor of 2.0 (Fig. 3b).

Theoretically, the QFT should yield a consistent $a_{\mathrm{p}}$ for any given sample, regardless of the volume filtered. In practice, however, slightly different values are obtained with variable sample loading. We evaluated relative standard error of $a_{\mathrm{p}}$ among different volumes filtered for each sample from each trial of the three microcosm experiments plus the Lake Nockamixon samples $(n=33)$. The Roesler approach yielded
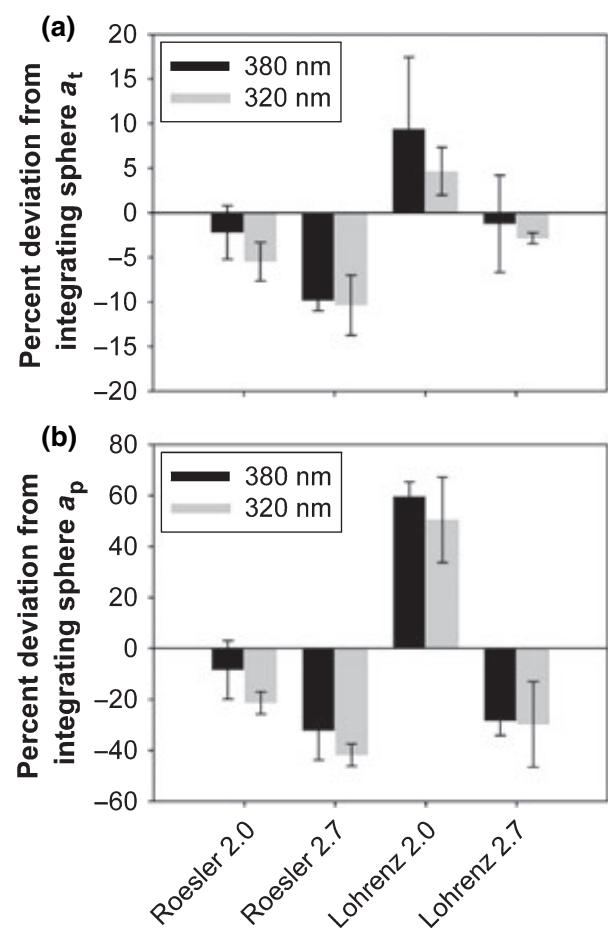

Figure 3. (a) Percentage deviation of $a_{\mathrm{t}}$ calculated with each of the four models using the QFT from $a_{\mathrm{t}}$ measured using the integrating sphere. (b) Percentage deviation of $a_{\mathrm{p}}$ calculated using the QFT from $a_{\mathrm{p}}$ back calculated from $a_{\mathrm{t}}$ measured using the integrating sphere. Values represent averages of two analytical replicates of two distinct samples, which differ in suspended sediment concentration by a factor of 2. Error bars represent standard error. 


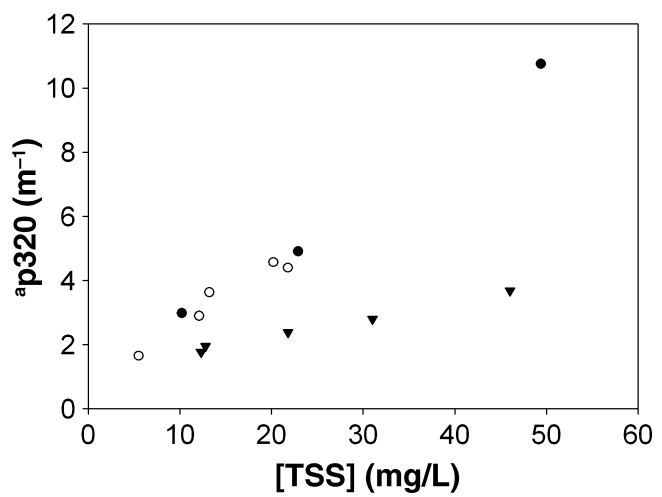

Figure 4. Each experiment shows a distinct trend of particulate absorption as a function of total suspended solid concentration ([TSS]). Differences could be attributed to seasonal changes in optical properties of particulates contributed from the catchment or location, as each of the experiments utilized water samples mixed proportionately from different parts of the Lehigh River watershed.

a relative standard error of $0.015(380 \mathrm{~nm})$ and 0.020 $(320 \mathrm{~nm})$. In contrast, the Lohrenz approach yielded relative standard errors that were more than twice those of Roesler ( 0.032 and 0.047 for 380 and $320 \mathrm{~nm}$, respectively).

Considering the four primary selection criteria, we chose the Roesler method, with a $\beta$ of 2.0 , as the most reliable and accurate model for estimating attenuation of UVR in stream water containing suspended particles. The rationale behind this choice is outlined in the Discussion section below. All relevant optical measurements presented from this point forward, including field-testing of the method and analysis of particulate optical quality, are consistent with the application of this model.

Total absorption coefficients for water samples taken from microcosm experiments ranged from 0.9-20.2, 1.7-30.4, 2.4-36.9 and 3.3-44.8 $\mathrm{m}^{-1}$ for wavelengths $380,340,320$ and $305 \mathrm{~nm}$, respectively. Field replicates yielded a coefficient of variation below 3\% for all UVR wavelengths, demonstrating the reduction in variance with the laboratory method. The average coefficient of variation among volume filtered replicates was $5 \%$.

When the particulate absorption data are pooled for all three microcosm experiments, the relationship between $a_{\mathrm{p} 320}$ and TSS is poor $\left(r^{2}=0.44\right)$. Individually however, the three experiments showed relatively consistent increases in particulate absorption per unit of TSS, but the trend differed for each experiment (Fig. 4). Particle-specific absorption ( $\left.a_{\mathrm{p} 320} /[\mathrm{TSS}]\right)$ for experiments 1,2 and 3 were $0.67( \pm 0.07), 0.11( \pm 0.03)$ and $0.12( \pm 0.02)$, respectively. Stepwise regression analysis indicates that bulk percentage POC does not account for the scatter observed in the relationship between $a_{\mathrm{p} 320}$ and [TSS] $\left(r^{2}\right.$ for $\%$ POC and residuals of TSS versus $a_{\mathrm{p} 320}=0.046$ ). Rather, it appears that particulate material collected from very similar watersheds can exhibit different optical quality at different times.

Comparison of $K_{\mathrm{d}}$ estimates made in situ with those made in the laboratory using the spectrophotometer

To field test the precision of the QFT, in situ measurements of $K_{\mathrm{dUV}}$ at 18 locations in the main stem and major tributaries of

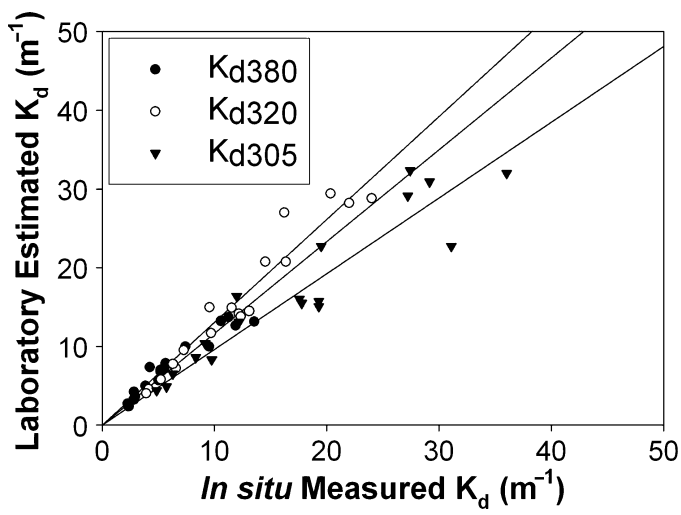

Figure 5. Regression of $K_{\mathrm{d}}$ values calculated using the QFT (Roesler 2.0 equations and associated $\mu$ values) to $K_{\mathrm{d}}$ measured in situ at 18 different locations along the main trunk and major tributaries of the Lehigh River. Regression slopes, forced through the origin, were 1.17, $1.31,0.96$ for 380,320 and $305 \mathrm{~nm}$, respectively.

the Lehigh River were compared to laboratory estimates of $K_{\mathrm{duv}}$. Diffuse attenuation coefficients determined in the laboratory ranged from $2.4-13.7 \mathrm{~m}^{-1}(380 \mathrm{~nm}), 4.0-29.4 \mathrm{~m}^{-1}$ $(320 \mathrm{~nm})$ and $4.4-32.3 \mathrm{~m}^{-1}(305 \mathrm{~nm}) . K_{\mathrm{d}}$ values measured in situ ranged from $2.3-13.5 \mathrm{~m}^{-1}(380 \mathrm{~nm}), 4.1-24 \mathrm{~m}^{-1}(320 \mathrm{~nm})$ and $5.7-36.0 \mathrm{~m}^{-1}(305 \mathrm{~nm})$. On average, $K_{\mathrm{d}}$ values estimated in the laboratory overestimated in situ values for $380 \mathrm{~nm}$ $(17 \%)$ and $320 \mathrm{~nm}(31 \%)$ but underestimated in situ measurements by $4 \%$ at $305 \mathrm{~nm}$ (Fig. 5). The attenuation values for these 18 sites suggest that $z_{1 \%}$ depths for the most damaging UVB radiation $(305 \mathrm{~nm})$ range between 0.81 and $0.13 \mathrm{~m}$. This implies that significant portions of the benthic community may be exposed to damaging levels of UVR.

\section{DISCUSSION}

\section{Development and testing of the filter pad method}

The goal of this study was to develop and calibrate a method by which laboratory measurements of dissolved and particulate absorption could be used for estimating diffuse attenuation coefficients. The most formidable challenge in generating such an attenuation model was estimating the contribution from suspended particles. In this study, four criteria were considered in selecting the optimal model, of the four tested, for calculating the particulate absorption coefficient. It is difficult to properly weight each of the different selection criteria in terms of relevance. Qualitatively, we can assert that the most important consideration is the slope and $r^{2}$ value of the regression of laboratory-estimated versus microcosmmeasured $K_{\mathrm{d}}$ values. The Lohrenz 2.0 method yields a substantially lower $r^{2}$ value compared with the rest. It is difficult to discriminate among models simply based on this regression analysis because the margin of error is reduced because we calculated the $\mu$ factor as a free parameter for each of the calculation methods, which was necessary because of the dynamic and poorly constrained nature of $\mu$. We chose the variation of $a_{\mathrm{p}}$ among volumes filtered as the next most relevant criterion. This parameter is important because it quantifies the model's sensitivity to filter loading. Although we strongly suggest measuring absorption for at least two different volumes for each sample, the error associated with 
this problem is minimized by selecting a method that yields consistent results. The Lohrenz equations were designed to minimize this problem for marine samples. Our data indicate that under low-filter-loading conditions the Lohrenz approach is marginally better at accounting for differences among volumes filtered, but under even moderately high filter loading with fluvial sediments, the Lohrenz approach performs very poorly. The integrating sphere comparison is another important selection criterion. The Lohrenz 2.7 and Roesler 2.0 models performed significantly better than the Lohrenz 2.0 and Roesler 2.7 models (Fig. 3). This was consistent with the $\beta$ values originally recommended by each of the authors, respectively $(13,14)$. Our rationale for testing alternate $\beta$ values for each of the equations was based on the notion that the optical properties of particulate material found in streams (typically of mineral origin) may differ substantially from that found in marine settings (typically organic), where both models were initially developed. Lastly, because of the errors associated with the calculation and the theoretical range of natural variability, the least relevant selection criterion is the $\mu$ factor. We simply evaluated whether or not the $\mu$ factors calculated from each of the methods were reasonable. Both Lohrenz approaches produced $\mu_{305}$ that equaled or exceeded 1.0 , which is technically unreasonable. However, considering the error associated with measuring attenuation of $305 \mathrm{~nm}$ light and considering that each $\mu$ factor is an average of 15 experiments, we considered only the Lohrenz 2.0 model as grossly overestimating the $\mu$ factor.

Considering our selection criteria for the optimal model, the Lohrenz 2.0 model was easily eliminated. The $r^{2}$ values of the calculated versus measured $K_{\mathrm{d}}$ were the lowest of the four models and the $\mu$ values generated were unacceptably high. The Lohrenz 2.0 model also had the highest standard deviation of $a_{\mathrm{p}}$ among volumes filtered and it performed poorly in the integrating sphere comparison. The Roesler 2.7 model can also be eliminated because it performed poorly in the integrating sphere comparison and did not perform particularly well in any of the other selection categories. The Lohrenz 2.7 model and Roesler 2.0 model each performed well for most of the tests, but the error of $a_{\mathrm{p}}$ calculated among different volumes filtered eliminates the Lohrenz 2.7 model from consideration. Thus, the Roesler model with a $\beta$ factor of 2.0 was selected as the most consistent and reliable method for estimating attenuation of stream water containing suspended particles from laboratory measurements of dissolved and particulate absorption.

Empirically derived models are most relevant to the field site where they are generated. The optical properties of particles are controlled by numerous environmental factors, including climate, parent material mineralogy and soil organic content. It is possible that the scattering and absorptive properties of particulates may vary for watersheds in which these deterministic factors differ substantially from the Lehigh River. Sutherland (18) demonstrated that significant variability exists in the organic matter content of fluvial sediments among streams and even among different grain sizes within the same stream. A survey of absorptive qualities of particulate material derived from different climatic and physiographic settings would be helpful in determining the magnitude of this variability. Such a survey will reduce uncertainty in extrapolation of the model proposed here to other systems and bring much needed constraints on the role that particles play in attenuation of UVR.

There are two types of error associated with using the QFT for estimating the particulate absorption coefficient and diffuse attenuation coefficient. The first is methodological and can be minimized with proper, standardized laboratory procedures. We recommend the use of a GF/A reference filter, the use of non-ashed GF/F filters for sample measurement and reasonable ranges for particle loading contribute to minimizing methodological error. The other source of error is associated with the theoretical conversion of beam absorption measured on a filter pad to attenuation of diffuse light in a water column containing particles. This source of error is related to the path length amplification in the spectrophotometer (corrected for by the $\beta$ factor), differences associated with the structure of the incident light field (corrected for by the $\mu$ factor) and the lack of accountability of scattering in the spectrophotometer with this method, which is designed to measure transmittance and assumes negligible scattering and constant reflectance. There are several options for future minimization of these sources of error.

The $\mu$ factor, which adjusts the beam absorption coefficient for diffuse light conditions, is likely the largest single source of error in estimating diffuse attenuation coefficients from laboratory measurements of absorption. The angular distribution of the incident light field can be affected by many variables, such as solar zenith angle, cloud density and thickness, atmospheric composition and canopy cover. The $\mu$ factor can be further modified by the relative intensity of absorption versus scattering in the water column and by depth. Although these can influence the penetration of UVR through the water column, the information required to properly model this phenomenon is not easily obtained with enough resolution to be useful. Moreover, for long-term studies, these factors likely average out and the variability is not likely to be of firstorder ecological importance. The variability of $\mu$ for each trial in our experiments was considerable as indicated by the standard errors shown in Table 4 , yet the average of $\mu$ seemed a reasonable estimate and is consistent with other published values. The error associated with the $\mu$ correction is most apparent in our field comparison, where estimates of $K_{\mathrm{d} 380}$ are $16 \%$ higher than measured values, whereas $K_{\mathrm{d} 305}$ was underestimated by $4 \%$. This is possibly due to the error in field measurements associated with the spectral change in the angular distribution of the light field as it passes through the riparian canopy. Two other potential sources of error include differences in particle absorption and errors associated with the $\mu$ values we have empirically derived. More work is obviously needed to better constrain this phenomenon. Conversion of an inherent optical property $\left(a_{\mathrm{t}}\right)$ to an apparent optical property $\left(K_{\mathrm{d}}\right)$ that is sensitive to many environmental variables makes it difficult to generate a meaningful total uncertainty for the method applied in the myriad of possible conditions. In consideration of this, we suggest that this model is best applied in locations with open canopy or where the effects of canopy can be accounted for (24). Furthermore, development of multiple alternative $\mu$ values to be used under different sky and/or canopy conditions may improve estimate precision.

Measuring particulate absorption on a filter pad also contributes to estimate error. Path length amplification occurs 
as a result of multiple scattering as light passes through the filter/sample matrix. Empirical quantification of this phenomenon has had limited success. The first issue to be addressed is whether or not $\beta$ should be a unique, fixed value. For the same reasons discussed in Roesler (13) and Lohrenz (14), we have chosen to take $\beta$ to be a fixed value. The theoretical rationale established by Roesler (13), which concludes that the $\beta$ value should be 2.0 , assumes that scattering is dominated by the filter itself and, thus, any additional scattering by the particles retained on the filter is negligible. More importantly, some unquantified portion of the error associated with measuring particulate absorption on a filter pad is due to the lack of accountability for backscattering in the spectrophotometer, which undoubtedly occurs, and may vary according to particulate material composition. The magnitude of this phenomenon likely varies with particulate composition and cannot simply be accounted for by a long-wavelength null point correction. The most reliable solution to this problem is to measure both, transmittance and reflectance as suggested by Tassan and Ferrari (21). Lohrenz et al. (25) confirmed that the transmittance-reflectance procedure significantly reduces error when comparing particulate absorption coefficients determined on a $\mathrm{GF} / \mathrm{F}$ filter with particulate absorption determined in suspension using an integrating sphere. The primary problem with this method is one of user accessibility. Few ecological research laboratories are equipped with the instrumentation needed to measure transmittance and reflectance on each sample. Currently without any other method to overcome this problem, the transmittance-reflectance method is not likely to be widely applicable for most studies primarily focused on ecological problems.

The study presented here is the first rigorous attempt at developing an empirical, field-tested model for estimating diffuse attenuation coefficients of UVR in lotic systems from laboratory measurements of dissolved and particulate absorption. Attempts were made to minimize various sources of error, while maintaining a methodology that is practical for all laboratories involved in UVR research. Application of this model will prove useful in estimating exposure of stream organisms to UVR.

\section{Potential applications of the method}

Attenuation depths for damaging UVB radiation in our 18 stream sites suggest that UVR may play an important role in structuring benthic communities. Exposure to UVR in streams is a function of the incoming irradiance, optical properties of the water, habitat characteristics and behavior of the target organism or community (26-28). Environmental change at local, regional and global scales, often exacerbated by human activity (5), has altered and will inevitably continue to alter the optical environment in streams at each of those spatial scales (29).

Changes in catchment land use will likely continue to affect stream optical environments both directly, by reducing riparian canopy cover and changing stream channel morphometry or indirectly, by changing the flux or optical quality of CDOM and/or sediment contributed to the channel. Such changes can take place over very short periods of time and vary substantially over short spatial distances (30-32). Very few studies have documented the influence of such changes on the penetration of UVR in streams. Because of the nature of land use change, this is a formidable challenge for stream ecologists.

Much work remains in documenting UVR transparency and variability in optical environments within and among streams. In a survey of 32 streams in northern Michigan, Frost et al. (33) documented a wide range of $1 \%$ transmission depths (2-45 cm for UVB and $6-103 \mathrm{~cm}$ for UVA). In the same region, Frost et al. (33) demonstrated nearly an order of magnitude difference in UVB flux within a $20 \mathrm{~m}$ stretch of stream and emphasized that canopy may play a large role in determining the UVR exposure in highly transparent streams with variable canopy cover.

On daily and even much shorter timescales, temperature, light intensity and water depth fluctuate at any given point in a stream channel. Storm events increase suspended sediment concentration, cause channel bed scour and move benthic substrate. Although UVR is most likely unimportant relative to other stresses during such events, gross restructuring of the bed morphology does reset conditions for niche competition after flow has abated and UVR could play an important role under these more transparent conditions. To date, we know of no studies that have investigated the importance of habitat complexity and light on microhabitat selection at the submeter scale in lotic systems. The QFT is well suited to help address such questions as it affords the ability to process large sample batches, especially in conjunction with a repetitive auto-sampler device, to estimate the benthic UVR flux in multiple locations simultaneously, at multiple points throughout a storm hydrograph.

Acknowledgement-This work was funded by EPA STAR grant 829642 to D. P. M.

\section{REFERENCES}

1. Williamson, C. E. (1995) What role does UV-B radiation play in freshwater ecosystems? Limnol. Oceanogr. 40, 386-392.

2. Kelly, D. J., M. L. Bothwell and D. W. Schindler (2003) Effects of solar ultraviolet radiation on stream benthic communities: An intersite comparison. Ecology 84, 2724-2740.

3. Hargreaves, B. R. (2003) Water column optics and penetration of UVR. In: UV Effects in Aquatic Organisms and Ecosystems (Edited by E. W. Helbling and H. E. Zagarese), pp. 59-105. Comprehensive Series in Photochemical and Photobiological Sciences, Royal Society of Chemistry, Cambridge, UK.

4. Naiman, R. J., J. J. Magnuson, D. M. McKnight and J. A. Stanford (1995) The Freshwater Imperative. Island Press, Washington, D.C.

5. Vitousek, P. M., H. A. Mooney, J. Lubchenco and J. M. Melillo (1997) Human domination of earth's ecosystems. Science 277, 494-499.

6. Schindler, D. W. (2001) The cumulative effects of climate warming and other human stresses on Canadian freshwaters in the new millennium. Can. J. Fish. Aquat. Sci. 58, 18-29.

7. Schindler, D. W., P. J. Curtis, B. R. Parker and M. P. Stainton (1996) Consequences of climate warming and lake acidification for UV-B penetration in North American boreal lakes. Nature 379, 705-708.

8. Madronich, S., R. L. McKenzie, L. O. Bjorn and M. M. Caldwell (1998) Changes in biologically active ultraviolet radiation reaching the Earth's surface. J. Photochem. Photobiol. B 46, 5-19.

9. Bothwell, M. L., D. M. J. Sherbot and C. M. Pollock (1994) Ecosystem response to solar ultraviolet-B radiation: Influence of trophic level interactions. Science 265, 97-100.

10. Watkins, E. M., D. W. Schindler, M. A. Turner and D. Findlay (2001) Effects of solar ultraviolet radiation on epilithic metabolism, 
nutrient and community composition in a clear-water boreal lake. Can. J. Fish. Aquat. Sci. 58, 2059-2070.

11. Palen, W. J., D. E. Schindler, M. J. Adams, C. A. Pearl, R. B. Bury and S. A. Diamond (2002) Optical characteristics of natural waters protect amphibians from UV-B in the U.S. Pacific Northwest. Ecology 83, 2951-2957.

12. Mitchell, B. G. (1990) Algorithms for determining the absorption coefficient of aquatic particulates using the quantitative filter technique (QFT). In Ocean Optics 10, Proc. SPIE 1302, pp. 137-148.

13. Roesler, C. S. (1998) Theoretical and experimental approaches to improve the accuracy of particulate absorption coefficients derived from the quantitative filter technique. Limnol. Oceanogr. 43, 16491660 .

14. Lohrenz, S. E. (2000) A novel theoretical approach to correct for pathlength amplification and variable sampling loading in measurements of particulate spectral absorption by the quantitative filter technique. J. Plankton Res. 22, 639-657.

15. Ayoub, L. M., B. R. Hargreaves and D. P. Morris (1996) UVR attenuation in lakes: Relative contribution of dissolved and particulate material. SPIE Ocean Optics XIII, 2963, 338-343.

16. Belzile, C., W. F. Vincent and M. Kumagai (2002) Contribution of absorption and scattering to the attenuation of UV and photosynthetically available radiation in Lake Biwa. Limnol. Oceanogr. 47, 95-107.

17. Hargreaves, B. R., S. F. Girdner, M. W. Buktenica, R. W. Collier, E. Urbach and G. L. Larson (2007) Ultraviolet radiation and biooptics in Crater Lake, Oregon. Hydrobiologia 574, 107-140.

18. Sutherland, R. A. (1998) Loss-on-ignition estimates of organic matter and relationships to organic carbon in fluvial bed sediments. Hydrobiologia 389, 153-167.

19. Kirk, J. T. O., B. R. Hargreaves, D. P. Morris, R. Coffin, B. David, D. Frederickson, D. Karentz, D. Lean, M. Lesser, S. Madronich, J. H. Morrow, N. Nelson and N. Scully (1994) Measurement of UV-B radiation in two freshwater lakes: An instrument intercomparison. Arch. Hydrobiol. Beih. Ergbn. Limnol. 43, 71-99.

20. Pope, R. M. and E. S. Fry (1997) Absorption spectrum (380$700 \mathrm{~nm}$ ) of pure water. II. Integrating cavity measurements. Appl. Opt. 36, 8710-8723.

21. Tassan, S. and G. M. Ferrari (1995) An alternative approach to absorption measurements of aquatic particles retained on filters. Limnol. Oceanogr. 40, 1358-1368.

22. Tassan, S. and G. M. Ferrari (1998) Measurements of light absorption by aquatic particles retained on filters: Determination absorption by aquatic particles retained on filters: Determination of the optical path length amplification by the 'transmittancereflectance' method. J. Plankton Res. 20, 1699-1709.

23. Tassan, S. and G. M. Ferrari (2002) A sensitivity analysis of the 'Transmittance-Reflectance' method for measuring light absorption by aquatic particles. J. Plankton Res. 24, 757-774.

24. Grant, R. H., G. M. Heisler and W. Gao (2002) Estimation of pedestrian level UV exposure under trees. Photochem. Photobiol. 75, 369-376.

25. Lohrenz, S. E., A. D. Weidemann and M. Tuel (2003) Phytoplankton spectral absorption as influenced by community size structure and pigment composition. J. Plankton Res. 25, 35-61.

26. Xenopoulos, M. A. and D. W. Schindler (2001) Physical factors determining ultraviolet flux into ecosystems. In Ecosystems, Evolution and UV Radiation (Edited by C. S. Cockell and A. R. Blaustein), pp. 36-62. Springer, New York.

27. Diamond, S. A., P. C. Trenham, M. J. Adams, B. R. Hossack, R. A. Knapp, S. L. Stark, D. Bradford, P. S. Corn, K. Czarnowski, P. D. Brooks, D. Fagre, B. Breen, N. E. Detenbeck and K. Tonnessen (2005) Estimated ultraviolet radiation doses in wetlands in six national parks. Ecosystems 8, 462-477.

28. Frost, P. C., A. Mack, J. H. Larson, S. D. Bridgham and G. A. Lamberti (2006) Environmental controls of UV-B radiation in forested streams of northern Michigan. Photochem. Photobiol. 82, 781-786.

29. UNEP EEAP Report (2006) Environmental effects of ozone depletion and its interactions with climate change: Progress Report 2005. Photochem. Photobiol. Sci. 5, 13-24.

30. Clark, J. J. and P. R. Wilcock (2000) Effects of landuse change on channel morphology in northeastern Puerto Rico. Geol. Soc. Am. Bull. 112, 1763-1777.

31. Kondolf, G. M., H. Piegay and N. Landon (2002) Channel response to increased and decreased bedload supply from land use change: Contrasts between two catchments. Geomorphology $\mathbf{4 5}$, $35-51$.

32. Galster, J. C., F. J. Pazzaglia, B. R. Hargreaves, D. P. Morris, S. C. Peters and R. N. Weisman (2006) Effects of urbanization on watershed hydrology: The scaling of discharge with drainage area. Geology 34, 713-716.

33. Frost, P. C., J. H. Larson, L. E. Kinsman, G. A. Lamberti and S. D. Bridgham (2005) Attenuation of ultraviolet radiation in streams of northern Michigan. J. North Am. Benthol. Soc. 24, 246255. 